Math. Model. Nat. Phenom.

Vol. 7, No. 1, 2012, pp. 3-28

DOI: $10.1051 / \mathrm{mmnp} / 20127102$

\title{
Cancer as Multifaceted Disease
}

\author{
A. Friedman* \\ Department of Mathematics, The Ohio State University, 43221 Columbus, OH USA
}

\begin{abstract}
Cancer has recently overtaken heart disease as the world's biggest killer. Cancer is initiated by gene mutations that result in local proliferation of abnormal cells and their migration to other parts of the human body, a process called metastasis. The metastasized cancer cells then interfere with the normal functions of the body, eventually leading to death. There are two hundred types of cancer, classified by their point of origin. Most of them share some common features, but they also have their specific character. In this article we review mathematical models of such common features and then proceed to describe models of specific cancer diseases.
\end{abstract}

Key words: tumor models, free boundary problems, hyperbolic equations, parabolic equations, Stokes equation

AMS subject classification: 35R35, 35Q92, 92C17, 92C50

\section{Introduction}

Cancers are classified by the tissue from which they arise and by the type of cells involved. For example, leukemia is a cancer of white blood cells, sarcoma is a cancer arising in muscles and connective tissue, and carcinoma is a cancer originating from epithelial cells, that is, the closely packed cells which align the internal cavities of the body. In this review we shall deal only with carcinomas, which is the most common type of cancer.

There are two ways by which a gene can become abnormal: (1) A stimulating gene becomes hyperactive, or upregulated; such an abnormal gene is called oncogene; and (2) An inhibitory gene becomes inactive, or downregulated; it is called a tumor suppressive gene, an example being the p53 gene which controls the initiation of the cell cycle.

\footnotetext{
*E-mail: afriedman@math.ohio-state.edu
} 
When one or several genes mutate and become abnormal, the cell and its progeny reproduce at a faster rate than normal cells.

Neoplasm, or tumor, is a growing mass of abnormal cells. As long as this mass remains clustered together and confined to the cavity, the tumor is said to be benign. If the tumor has emerged out of the cavity, by breaking through the basal membrane and then proliferating into the extracellular matrix, or stroma, then the tumor has become malignant, and we refer to it as cancer. When cancer cells invade into the blood stream or the lymphatic vessels, they may then be transported into another location, thus creating a secondary tumor; this process is called metastasis.

There are two hundred different types of cancer that can affect the human body. They differ by the location of the initial cancer and by the type of cells. At the same time there are common features shared by many types of cancer. For example, all growing solid tumors, such as carcinoma, require more oxygen and nutrients than is available in normal healthy tissue. They therefore secrete Vascular Endothelian Growth Factor (VEGF) in order to grow and attract blood vessels, a process called angiogenesis.

This review is divided into two parts. In the first part we describe mathematical models that have been used to describe general features of tumor growth, shared by most solid tumors, including carcinoma and sarcoma. In the second part we focus on several specific tumors/cancers to illustrate the multifaceted nature of the disease.

\section{Part I. Mathematical Models for Generic Solid Tumors}

In this part we consider mathematical models of non-specific solid tumors in a tissue. The models incorporate the constitutive nature of the tissue, and the need for growing tumors to attract blood vessels. We also describe a general multi-scale approach that involves cell cycle and incorporates non-specific genes mutation.

\section{Tissue Structure}

The tissue in which cells proliferate is called the Extracellular Matrix (ECM). It has a complicated structure, so most mathematical models make simplified assumptions on the ECM structure. We shall review three different approaches.

\subsection{Porous medium}

In this section, we assume that the tumor includes three types of cells: proliferating cells with (mass) density $p(x, t)$, quiescent cells with density $q(x, t)$, and dead cells with density $n(x, t)$.

Following [62], we assume that quiescent cells become proliferating at a rate $K_{P}(c)$ which depends on the concentration of nutrients $c$, and they become necrotic at death rate $K_{D}(c)$. We also assume that proliferating cells become quiescent at a rate $K_{Q}(c)$ and their death rate is $K_{A}(c)$. 
The density of proliferating cells is increasing at a rate $K_{B}(c)$. Finally, we assume that dead cells are removed from the tumor (by macrophages) at a constant rate $K_{R}$.

We also assume that all the cells are physically identical in volume and mass and that their density is constant throughout the tumor. Then

$$
p+q+n=\text { const. }=\theta \text {. }
$$

Due to proliferation and removal of cells, there is a continuous motion of cells within the tumor. We shall represent this movement by a velocity field $\mathbf{v}$. We can then write the conservation of mass law for the densities of the proliferating cells $p$, the quiescent cells $q$, and the dead cells $n$ within the tumor region $\Omega(t)$ in the following form:

$$
\begin{gathered}
\frac{\partial p}{\partial t}+\operatorname{div}(p \mathbf{v})=\left[K_{B}(c)-K_{Q}(c)-K_{A}(c)\right] p+K_{P}(c) q \\
\frac{\partial q}{\partial t}+\operatorname{div}(q \mathbf{v})=K_{Q}(c) p-\left[K_{P}(c)+K_{D}(c)\right] q \\
\frac{\partial n}{\partial t}+\operatorname{div}(n \mathbf{v})=K_{A}(c) p+K_{D}(c) q-K_{R} n
\end{gathered}
$$

The tumor tissue will be treated as a porous medium and the moving cells as fluid flow. In a porous medium, the velocity $\mathbf{v}$ of fluid flow is related to the fluid pressure $\sigma$ by means of Darcy's law,

$$
\mathbf{v}=-\nabla \sigma
$$

If we add equations (2.2)-(2.4) and use (2.1), we get

$$
\theta \operatorname{div} \mathbf{v}=K_{B}(c) p-K_{R} n
$$

and we may replace (2.4) by (2.6).

We assume that the nutrient concentration satisfies the diffusion equation

$$
\epsilon_{0} \frac{\partial c}{\partial t}=\Delta c-\lambda(p+q) \text { in } \Omega(t)
$$

Eliminating $n$ from (2.6) by (2.1) and taking, for simplicity, $\theta=1$, and recalling (2.5), we obtain, in addition to (2.7), the following equations:

$$
\begin{gathered}
\frac{\partial p}{\partial t}-\nabla \sigma \cdot \nabla p=f(c, p, q) \text { in } \Omega(t), t>0 \\
\frac{\partial q}{\partial t}-\nabla \sigma \cdot \nabla q=g(c, p, q) \text { in } \Omega(t), t>0 \\
\Delta \sigma=-h(c, p, q) \text { in } \Omega(t), t>0
\end{gathered}
$$


where

$$
\begin{array}{r}
f(c, p, q)=\left[K_{B}(c)-K_{Q}(c)-K_{A}(c)\right] p+K_{P}(c) q-h(c, p, q) p \\
g(c, p, q)=K_{Q}(c) p-\left[K_{P}(c)+K_{D}(c)\right] q-h(c, p, q) q \\
h(c, p, q)=-K_{R}+\left[K_{B}(c)+K_{R}\right] p+K_{R} q .
\end{array}
$$

We denote the boundary of $\Omega(t)$ by $\Gamma(t)$ and impose the following boundary conditions:

$$
\begin{gathered}
c=\bar{c} \text { on } \Gamma(t), t>0, \\
\sigma=\gamma \kappa \text { on } \Gamma(t), t>0,
\end{gathered}
$$

where $\bar{c}$ is a constant, $\gamma$ is a surface tension coefficient representing the cell-to-cell adhesion, and $\kappa$ is the mean curvature $\left(\kappa=\frac{1}{R}\right.$ if $\Omega(\mathrm{t})$ is a ball of radius $R$ ). The boundary of the tumor varies in time; it is called a 'free boundary'. We assume that its normal velocity $V_{n}$ is the same as the normal velocity $\mathbf{v} \cdot \mathbf{n}$ of the cells in the outward normal direction $\mathbf{n}$, i.e.,

$$
\frac{\partial \sigma}{\partial n}=-V_{n} \text { on } \Gamma(t), t>0
$$

The system (2.7)-(2.13) with initial conditions on $c, p$ and $q$ was investigated mathematically. Local existence and uniqueness of a solution with prescribed initial data was proved in [6] [11]. Under radially symmetric data, global existence of a radially symmetric solution was proved and asymptotic estimates were derived on the free boundary $\Gamma(t)=\{r=R(t)\}$ as $t \rightarrow \infty$ [15]. There are only partial results on the existence and uniqueness of a radially symmetric stationary solution and its asymptotic stability [12] [14]. However, in the case of only one population of cells (i.e., $p \equiv 1, q \equiv n \equiv 0)$ it was proved that there exists a unique radially symmetric stationary solution [36]; it is asymptotically stable for all $\gamma<\gamma_{*}$ (for some $\gamma_{*}>0$ ) but not for $\gamma>\gamma_{*}$ [5] [29]; see also [70]. Furthermore, there are symmetry-breaking bifurcation branches of solutions initiating from points $\gamma_{2}, \gamma_{3}, \ldots, \gamma_{n}, \ldots$, where $\gamma_{2} \geq \gamma_{*}$ [20] [28] [32] [37].

\subsection{Fluid tissue}

In cancer which initiates in the mammary gland or in the brain it is more appropriate to assume that the tissue is fluid-like rather than a porous medium, and to use the Stokes equation instead of the Darcy's law. In this case the relation between the velocity $\mathbf{v}$ and the pressure $\sigma$ is given by

$$
\begin{gathered}
-\nu \nabla^{2} \mathbf{v}+\nabla \sigma=\mathbf{f}, \\
\operatorname{div} \mathbf{v}=\mathrm{g}
\end{gathered}
$$

where $\mathbf{f}=-\frac{\nu}{3} \nabla g$, and the boundary condition (2.12) is replaced by

$$
T(\mathbf{v}, \sigma) \mathbf{n}=-\gamma \kappa \mathbf{n}
$$

where

$$
T(\mathbf{v}, \sigma)=\nu\left(\nabla \mathbf{v}+\nabla \mathbf{v}^{\mathrm{T}}\right)-\left(\sigma+\frac{2 \nu}{3} \operatorname{div} \mathbf{v}\right) \mathrm{I},
$$


$\nu$ is the viscosity coefficient, $I$ is the unit matrix, and $g$ is the proliferation rate, given by the righthand side of (2.6). Existence and uniqueness of a solution with prescribed initial data was proved in [25]. In the case of one population of cells $(p \equiv 1, q \equiv n \equiv 0)$ the existence of a unique stationary solution with radially symmetric free boundary was proved in [25] and its asymptotic stability if $\gamma<\gamma_{* *}$ (for some $\gamma_{* *}>0$ ), but not if $\gamma>\gamma_{* *}$, was proved in [30]. As in the case of porous medium, here too there exists a sequence of symmetry-breaking bifurcation branches of solutions [31].

\subsection{ECM density}

The ECM structure is a complex network of protein fibers, mostly collagens. A common simplified approach to model the ECM is by the concentration $\rho$ of these fibers. The fibers are typically produced by fibroblast cells. Cancer cells (with density $n$ ) move within the ECM by breaking down some of the fibers of the ECM. They do so by degrading the matrix by means of an enzyme called Matrix Metalloproteinase (MMP). Hence $\rho$ satisfies the differential equation

$$
\frac{\partial \rho}{\partial t}=-a_{1} P n+a_{2} f\left(1-\frac{\rho}{\rho_{*}}\right)
$$

where $P$ is the concentration of MMP, $\rho_{*}$ is the maximum ECM density, and $a_{i}$ are positive constants. Of course we need to provide also equations for $\mathrm{P}$ and $f$, but these equations will depend on the particular cancer and on its microenvironments.

Hybrid models for tumor cells migration in ECM were developed in [54].

\section{Avascular/Vascular tumors}

As a tumor grows it reaches a size where cells in the core do not get enough oxygen from the vascular system. At this point cancer cells begin to secrete Tumor Angiogenic Factor (TAF), such as VEGF, which stimulates endothelial cells to move out of existing capillaries, proliferate, and form new capillaries (a process called angiogenesis) that move toward the tumor. Mathematical models distinguish between avascular tumors (preangiogenesis) and vascular tumors. In modeling vascular tumors the process of angiogenesis is incorporated by adding additional differential equations to a particular cancer model.

There are quite a number of mathematical models of angiogenesis. We refer, in particular, to [57] [58], [60] and the references therein. A somewhat simple model, based on [58], [60], which includes the main ingredients from these papers, is as follows:

Set

$n=$ density of tumor cells,

$e=$ density of endothelial cells,

$a=$ concentration of TAF, 
$c=$ concentration of proteolytic enzyme,

$c_{a}=$ active forms of $\mathrm{c}$,

$f=$ concentrtion of fibronectin; these are the fibers that connect cells to collagen, thereby enabling cells to move in the tissue

Then

$$
\begin{gathered}
c_{a}=\frac{c}{1+\alpha_{0} f}, \\
\frac{\partial a}{\partial t}=D_{a} \nabla^{2} a+\alpha_{1} n-\frac{\alpha_{2} a}{1+\alpha_{3} a} e, \\
\frac{\partial c}{\partial t}=D_{c} \nabla^{2} c+\frac{\alpha_{2} a}{1+\alpha_{3} a} e-\alpha_{4} c \\
\frac{\partial f}{\partial t}=D_{f} \nabla^{2} f+\alpha_{5} f\left(1-\frac{f}{f_{0}}\right)-\frac{\alpha_{6} f}{1+\alpha_{7} f} c_{a}, \\
\frac{\partial e}{\partial t}=D_{e} \nabla \cdot\left[\nabla \ln \frac{e}{\tau\left(c_{a}, f\right)}\right]
\end{gathered}
$$

where

$$
\tau\left(c_{a}, f\right)=\left(\frac{c_{a}+\beta_{1}}{c_{a}+\beta_{2}}\right)^{\gamma_{1}}\left(\frac{f+\delta_{1}}{f+\delta_{2}}\right)^{\gamma_{2}}
$$

is a chemotactic function; the $\alpha_{i}, \beta_{i}, \delta_{i}$ are constants.

The dispersion coefficients $D_{c}, D_{f}$ are extremely small and, in fact, do not appear in [58] [60]. The PDE system (3.1)-(3.6) needs to be supplemented with boundary conditions and initial conditions.

The tumor cells near the tumor boundary clearly receive more oxygen and hence proliferate more vigorously than the cells in the core. In fact, most of the proliferation occurs in the outer shells of the tumor whereas the inner core tends to be necrotic. Such models have been considered in [9] [13] and the references therein.

\section{Multiscale approach}

Most mathematical models are based on differential equations for variables that are densities of cells and concentrations of proteins and other molecules. Since, however, cancer originates from gene mutations, models of a particular cancer may need to include the effect of genes mutation which occur at the microscopic level. Furthermore, the decision of a cell to proliferate or remain quiescent is made at a particular time point in the cell cycle, as is the decision of a cell whether to commit suicide (apoptosis) in case it detects a damage that may not be reparable. Thus, in addition to the time $t$, we may want to include the time $s$ of cell cycle, which is typically a few days. The cell cycle is divided into four phases: $S$ (for synthesis), $M$ (for mitosis), and gap phases 
$G_{1}$ and $G_{2}$. During the $S$ phase the DNA is replicated, that is, each chromosome is duplicated. During the mitosis phase, $M$, the nuclear membrane breaks down, sister chromatids are separated, new nuclear membranes are formed, and the cell divides into two daughter cells. $S$ and $M$ are separated by two gap phases, $G_{1}$ and $G_{2}$. The cell cycle is controlled at two check points, $R_{1}$, located near the end of $G_{1}$, and $R_{2}$ located in $G_{2}$.

At the check point $R_{1}$ the cell decides on one of three options: (i) to commit suicide (apoptosis) if it senses that it has been damaged beyond repair during the growth phase $G_{1}$; (ii) to go into a quiescent phase $G_{0}$ and stay there for a while, if the microenvironment is hypoxic or overpopulated with other cells; or (iii) to proceed to the $S$ phase. At $R_{2}$ the cells decide either to go into apoptosis if irreparable damage has occurred during the DNA replication, or to continue toward the $M$ phase. A cell remains in $G_{0}$ for a period of time, at the end of which it proceeds to the $S$ phase.

We describe a multiscale model in a simple case where the only cells are cancer cells with possible mutations in the set of genes $\gamma=\left(\gamma_{1}, \gamma_{2}, \ldots, \gamma_{\ell}\right)$. We shall show how equations (2.2) (2.4) are to be changed and replaced by a system of equations for cancer cells $p_{i}$ in different phases $i$.

We introduce the following notation:

$$
\begin{aligned}
& p_{1}\left(x, t, s_{1}\right)=\text { density of cells in phase } \mathrm{G}_{1}, 0 \leq \mathrm{s}_{1} \leq \mathrm{A}_{1} \\
& p_{2}\left(x, t, s_{2}\right)=\text { density of cells in phase } \mathrm{S}, 0 \leq \mathrm{s}_{2} \leq \mathrm{A}_{2} ; \\
& p_{3}\left(x, t, s_{3}\right)=\text { density of cells in phases } \mathrm{G}_{2} \text { and } \mathrm{M}, 0 \leq \mathrm{s}_{3} \leq \mathrm{A}_{3} \\
& p_{0}\left(x, t, s_{0}\right)=\text { density of cells in phase } \mathrm{G}_{0}, 0 \leq \mathrm{s}_{0} \leq \mathrm{A}_{0} \\
& p_{4}(x, t)=\text { density of dead cells. }
\end{aligned}
$$

The variable $x$ will vary in the tumor region $\Omega(t)$ in $\mathbb{R}^{3}$, with boundary $\Gamma(t)$.

We denote by $w(x, t)$ the oxygen concentration and by $Q(x, t)$ the combined density of live cells in phases $G_{1}, S, G_{2}, M$ and $G_{0}$. Then, by conservation of mass,

$$
\begin{gathered}
\frac{\partial p_{i}}{\partial t}+\frac{\partial p_{i}}{\partial s_{i}}+\operatorname{div}\left(p_{i} \mathbf{v}\right)=\lambda_{i}(w) p_{i} \text { for } 0<\mathrm{s}_{\mathrm{i}}<\mathrm{A}_{\mathrm{i}}(\mathrm{i}=1,2,3), \\
\frac{\partial p_{0}}{\partial t}+\frac{\partial p_{0}}{\partial s_{0}}+\operatorname{div}\left(p_{0} \mathbf{v}\right)=-\lambda_{0} p_{0} \text { for } 0<\mathrm{s}_{0}<\mathrm{A}_{0}, \\
\frac{\partial p_{4}}{\partial t}+\operatorname{div}\left(p_{4} \mathbf{v}\right)=\mu_{1} p_{1}\left(x, t, A_{1}\right)+\mu_{2} p_{2}\left(x, t, A_{2}\right)-\lambda_{4} p_{4}
\end{gathered}
$$

where $\lambda_{i}(w)$ are growth rates, and $\mu_{i}=\mu_{i}(\gamma)$.

Since the density of cells does not change during the time of replication,

$$
p_{1}(x, t, 0)=p_{3}\left(x, t, A_{3}\right) .
$$

In the following equation (4.5) the first term on the right-hand side represents the cell's decision at check point $R_{1}$ whether to go to apoptosis $\left(\mu_{1}\right)$, to quiescent phase, or to proceed to $S$; the decision depends on the environment $(w, Q)$ and on the state of the genes in $\gamma$. The last term in (4.5) represents cells that moved from $G_{0}$ to $S$,

$$
p_{2}(x, t, 0)=\left(1-\mu_{1}-K(w(x, t), Q(x, t) ; \gamma)\right) p_{1}\left(x, t, A_{1}\right)+p_{0}\left(x, t, A_{0}\right) .
$$


The decision at $R_{2}$, whether to go to apoptosis, is expressed by the equation

$$
p_{3}(x, t, 0)=\left(1-\mu_{2}\right) p_{2}\left(x, t, A_{2}\right),
$$

and the quiescence phase begins with

$$
p_{0}(x, t, 0)=K(w(x, t), Q(x, t), \gamma) p_{1}\left(x, t, A_{1}\right) .
$$

The system (4.1)-(4.7) is still incomplete since we did not specify the velocity $\mathbf{v}$. To do that we introduce the quantities

$$
\begin{aligned}
& Q_{i}(x, t)=\int_{0}^{A_{i}} p_{i}\left(x, t, s_{i}\right) d s_{i}(0 \leq i \leq 3), Q_{4}(x, t)=p_{4}(x, t), \\
& \vec{Q}(x, t)=\left\{Q_{i}\right\}_{i=0}^{4},
\end{aligned}
$$

and note that

$$
Q(x, t)=\Sigma_{i=0}^{3} Q_{i}(x, t) .
$$

We integrate equations (4.1)-(4.2) over their respective $s_{i}$-intervals and combine the result together with (4.3). Using (4.4)-(4.7) we find that all the boundary terms cancel out, so that

$$
\sum_{i=0}^{4}\left[\frac{\partial Q_{i}}{\partial t}+\operatorname{div}\left(Q_{i} \vec{v}\right)\right]=\sum_{i=1}^{3} \lambda_{i}(w) Q_{i}-\lambda_{0} Q_{0}-\lambda_{4} Q_{4}
$$

If we now assume, as in (2.1), that

$$
\sum_{i=0}^{4} Q_{i}(x, t) \equiv \text { const. }=\theta,
$$

then (4.8) yields

$$
\theta \operatorname{div} \vec{v}=H(\vec{Q}, w)
$$

where

$$
H(\vec{Q}, w)=\sum_{i=1}^{3} \lambda_{i}(w) Q_{i}-\lambda_{0} Q_{0}-\lambda_{4} Q_{4} .
$$

Finally, the oxygen concentration satisfies a diffusion equation

$$
w_{t}-D_{w} \cdot \nabla^{2} w+\bar{\lambda} Q w=b
$$

where $\bar{\lambda}$ is a positive constant, and $b=0$ during the avascular stage of the tumor.

We can proceed to complete the model by assuming that the tissue either obeys Darcy's law (Section 2.1) or Stokes law (Section 2.2). These models were studies in [26] [27] where local existence and uniqueness was proved for any initial data and global existence was proved in the radially symmetric case. Some asymptotic estimates were derived in [33]. For other related multiscale models see [3] [50] [64] [65]. 


\section{Part II. Models for Specific Tumors}

Table 1 lists the most common cancers in the U.S.

\begin{tabular}{|l|c|c|}
\hline Cancer type & New cases & Deaths \\
\hline lung & 223,000 & 157,000 \\
colorectal & 103,000 & 51,000 \\
breast & 207,000 & 40,000 \\
prostate & 218,000 & 32,000 \\
pancreatic & 43,000 & 39,000 \\
leukemia & 43,000 & 25,000 \\
non-hodgkin lymphoma & 66,000 & 20,000 \\
liver & 24,000 & 19,000 \\
bladder & 71,000 & 15,000 \\
kidney & 58,000 & 13,000 \\
glioma & 22,000 & 13,000 \\
melanoma & 68,000 & 9,000 \\
\hline
\end{tabular}

Table 1. Estimated new cases and deaths in the U.S. for the year 2010, for most of the leading cancers.

Notice the discrepancy in new cases vs. deaths among the different cancer types. For example, the ratio between annual deaths to annual new cases for pancreatic cancer is $90 \%$, for glioma it is $59 \%$, while for prostate cancer it is only $15 \%$. This clearly suggests that each type of cancer has its own specific issues.

In this part we give four examples of specific cancers, each with its specific biological conditions and its corresponding mathematical model: breast cancer (early stage), colorectal cancer, glioma, and prostate cancer.

\section{Breast cancer (initial stage)}

There are many mathematical models of breast cancer, especially for ductal carcinoma in situ (DCIS), that is, before the cancer cells break out of the mammary gland and migrate into the stroma; see [21] [22] [23][24] and the references therein. Since early detection is a critical factor in successful treatment, we focus here on the initial proliferation of cancer cells.

It is well known that tumor and its microenvironment, or stroma, interact with each other and that this interaction plays a critical role in tumor initiation, growth, and metastasis. This interaction consists of complex relations between tumor cells, stromal cells such as fibroblasts, epithelial cells and immunocytes, the vascular system, the extracellular matrix, and cytokines secreted by the cells. Understanding these relationships may lead to new therapeutic approaches to cancer. 
Here we consider the interaction between tumor epithelial cells (TECs) and fibroblasts in vitro microenvironment. It is known that breast cancer risk is associated with increased levels of Epidermal Growth Factors (EGF) and Tumor Growth Factor- $\beta$ (TGF- $\beta$ ). TGF- $\beta$ is secreted by TECs, whereas EGF is produced by the fibroblasts. TGF- $\beta$ is also known to cause fibroblasts to transform into myofibroblasts, which increases their secretion of EGF. The signaling cascade

$$
\mathrm{TEC} \rightarrow \text { TGF- } \beta \rightarrow \text { fibroblasts (myofibroblasts) } \rightarrow \text { TEC }
$$

is autocatalytic, and this situation corresponds to early stage of breast tumor such as DCIS. In [55] a mathematical model was developed which corresponds to experiments done in a transwell, shown systematically in Figure 5.1.

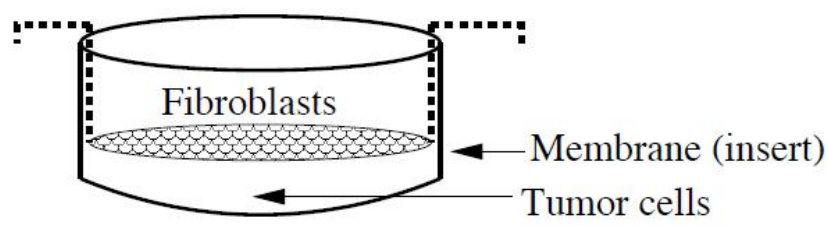

Figure 1: Structure of a transwell kit.

The main conclusions in [55] are that [(i) fibroblasts enhance proliferation of breast cancer cell lines, (ii) TEC population is sensitive to membrane permeability and to the transformation rate from fibroblasts to myofibroblasts, and (iii) interaction between TECs and fibroblasts promotes not only TEC proliferation but also the proliferation of fibroblasts/myofibroblasts and the transformation from fibroblasts to myofibroblasts. The model was in good agreement with experimental results reported in [55].

Based on this initial work, a mathematical model was developed in [35] [52] to determine in more detail the effect of the ECM of tumor cell migration. The experimental set-up is that of a Boyden Chamber Invasion Assay, shown in Figure 5.2; a simplified schematics is shown in figure 5.3.

The governing equations for these variables are:

$$
\begin{aligned}
\frac{\partial n}{\partial t}= & \nabla \cdot\left(D_{n} \nabla_{n}\right)-\nabla \cdot \underbrace{\left(\chi_{n} n \frac{\nabla E}{\sqrt{1+\left(|\nabla E| / \lambda_{E}\right)^{2}}}\right.}_{\text {chemotaxis }}+\underbrace{\left.\chi_{n}^{1} I_{S} n \frac{\nabla \rho}{\sqrt{1+\left(\left|\nabla_{\rho}\right| / \lambda_{\rho}\right)^{2}}}\right)}_{\text {haptotaxis }} \\
& +\underbrace{a_{11} \frac{E^{4}}{k_{E}^{4}+E^{4}} n\left(1-\frac{n}{n_{*}-a_{12} \rho I_{S}}\right)}_{\text {proliferation }} \text { in } \Omega_{+}, t>0,
\end{aligned}
$$




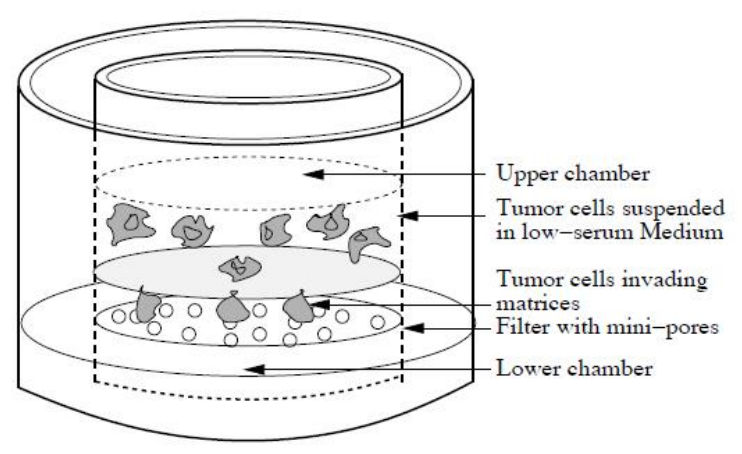

Figure 2: Illustration of a Boyden Chamber Invasion Assay that mimics tumor invasion in vivo

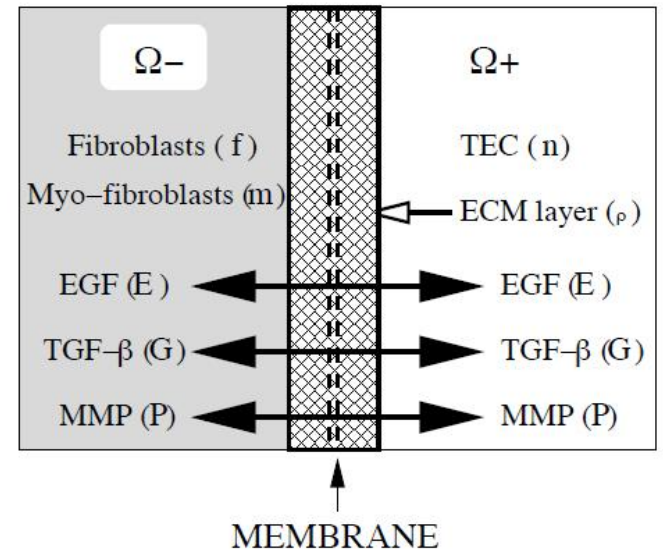

Figure 3: Schematics of an invasion Assay System: EGF $(E)$, TGF- $\beta(G)$ and MMP $(P)$ can cross the semi-permeable membrane, but the cells (TECs $(n)$, fibroblasts $(f)$, myofibroblasts $(m)$ ) may not cross it. Initially the TECs reside in the domain $\Omega_{+}$while fibroblasts and myofibroblasts are placed in the domain $\Omega_{-}$. An ECM layer with density $\rho$ surrounds the semi-permeable membrane (filter). 


$$
\begin{aligned}
& \frac{\partial f}{\partial t}=\nabla \cdot\left(D_{f} \nabla f\right)-\underbrace{a_{21} G f}_{f \rightarrow m}+\underbrace{a_{22} f}_{\text {proliferation }} \text { in } \Omega_{-}, t>0, \\
& \frac{\partial m}{\partial t}=\nabla \cdot\left(D_{m} \nabla m\right)-\underbrace{\nabla \cdot\left(\chi_{m} m \frac{\nabla G}{\sqrt{1+\left(|\nabla G| / \lambda_{G}\right)^{2}}}\right)}_{\text {chemotaxis }} \\
& +\underbrace{a_{21} G f}_{f \rightarrow m}+\underbrace{a_{31} m}_{\text {proliferation }} \text { in } \Omega_{-}, t>0, \\
& \frac{\partial \rho}{\partial t}=-\underbrace{a_{41} P n}_{\text {degradation }}+\underbrace{\left(a_{42} f+a_{43} m\right)\left(1-\frac{\rho}{\rho_{*}}\right)}_{\text {release/reconstructiion }} \text { in } S, t>0, \\
& \frac{\partial E}{\partial t}=\nabla \cdot\left(D_{E} \nabla E\right)+\underbrace{I_{\Omega_{-}}\left(a_{51} f+a_{52} m\right)}_{\text {production }}-\underbrace{a_{53} E}_{\text {decay }} \text { in } \Omega_{*}, t>0, \\
& \frac{\partial G}{\partial t}=\nabla \cdot\left(D_{G} \nabla G\right)+\underbrace{a_{61} I_{\Omega_{+}} n}_{\text {production }}-\underbrace{a_{62} G}_{\text {decay }} \text { in } \Omega_{*}, t>0, \\
& \frac{\partial P}{\partial t}=\nabla \cdot\left(D_{P} \nabla P\right)+\underbrace{a_{71} I_{\Omega_{-}} m}_{\text {production }}-\underbrace{a_{72} P}_{\text {decay }} \text { in } \Omega_{*}, t>0,
\end{aligned}
$$

where $I_{S}$ is a characteristic function of the set $S$ where the ECM density $\rho$ is positive. The cytokines in equations (5.1)-(5.7) undergo diffusion, production and degradation. We refer to [52] for more details, including boundary conditions and the determination of the parameters.

Some of the conclusions of the model simulations are the following:

(i) Haptotaxis increases myofibroblasts secreted MMP and the migration of tumor cells

(ii) As a function of the initial ECM concentration $\rho_{0}$, the total TEC population displays an interesting behavior: it increases with $\rho_{0}$ as long as $\rho<\bar{\rho}_{0}\left(\bar{\rho}_{0}=6 \times 10^{-4}\right)$ and decreases with $\rho_{0}$ when $\rho_{0}>\bar{\rho}_{0}$. This behavior has been observed in experiments [4] [61].

In the special case where the pore size is large enough so that the membrane is permeable also to cells, the following additional conclusions were derived from the model:

(iii) an increase in the permeability $\gamma_{0}$ of the membrane will result in increased TEC population; this is a natural conclusion due to the fact that cells can then more easily migrate through the filter.

(iv) As the thickness of $\mu$ of the ECM layer increases, the total TEC population decreases as long as $\mu<\mu_{0}\left(\mu_{0}=0.3\right)$ and then it increases for $\mu>\mu_{0}$. This may be explained by "competition for space" which is undergoing within the ECM layer. This conclusion, however, needs to be validated by experiments. 


\section{Colorectal cancer}

When a small number of genes commonly mutated give rise to the initiation of a particular cancer, one may model the process by the multiscale model described in Section 4. This situation occurs in colorectal cancer with the mutations of the five genes APC, RAS, TGF- $\beta$, SMAD and p53 [64] [65]. The first four mutations affect the decision of cells at the first check point $R_{1}$; a mutation in p53 may result in a decision by an irreparably damaged cell, either at $R_{1}$ or at $R_{2}$ check points, not to commit to apoptosis.

Hypoxia is sensed by a network that includes TGF- $\beta$ and SMAD. When these genes are mutated the cell will become insensitive to hypoxia, i.e., to abnormally low levels of oxygen. Overpopulation signal is sensed by APC. If APC is mutated the cell may decide to go directly from $G_{1}$ to $S$ even if the microenvironment is overcrowded with cells.

We can use the model described in Section 4 to respond, in a theoretical way, to questions like:

(i) If mutations occur only in TGF- $\beta$ and SMAD, would this result in cancer?

(ii) If mutation occurs also in APC, would this result in cancer?

If only TGF- $\beta$ and SMAD are mutated, then we take the function $K$ in (4.5) to be independent of the oxygen concentration $w$, so that

$$
K=K\left(Q(x, t), \gamma_{1}\right) \text { where } \gamma_{1}=\mathrm{APC} \text { is active at normal/healthy level. }
$$

If also APC is mutated, then

$$
K(Q(x, t))=\text { const. }=\beta
$$

In the case of (6.1), we view $K\left(Q, \gamma_{1}\right)$ as a control function of the variable $Q$. The aim of this control is to keep the tissue volume within homeostatic bounds, so that the tumor remains benign.

To address mathematically the questions associated with (6.1), (6.2) we assume that the tumor is radially symmetric and denote its radius by $R(t)$. We can then reformulate the above questions in the following way:

What is the behavior of $R(t)$ in case

(i) $K(Q)=$ const. $=\beta$, i.e., the cells do not respond to overpopulation;

(ii) $K\left(Q, \gamma_{1}\right)$ is a 'very good' control function in the variable $Q$.

The following results were proved in [34]:

(i') Assume that

$$
1<\left(1-\mu_{1}\right)\left(1-\mu_{2}\right) e^{\lambda\left(A_{1}+A_{2}+A_{3}\right)}<e^{\lambda_{0} A_{0}}
$$

where $\lambda_{i}(w)=\lambda$ if $1 \leq i \leq 3$. The quantity $e^{\lambda\left(A_{1}+A_{2}+A_{3}\right)}$ represents the growth of cells in phases $G_{1}, G_{2}, G_{3}$, and the first inequality in (6.3) means that, despite apoptosis, the tumor is growing if we ignore the time the cells spend in quiescent phase. The second inequality means that if we increase the time cells spend (and necrose) in the quiescent phase $G_{0}$, then the tumor is shrinking. Thus the decision of the cells at check point $R_{1}$ is critical: it determines whether the tumor will grow or shrink. If $\beta$ is small then the rate of cells going into the quiescent phase is small and, consequently, the tumor will grow, whereas if $\beta$ is large then the tumor will shrink. Indeed, there exists a positive number $\beta^{*}, \beta^{*}<1-\mu_{1}$, such that if $\beta<\beta^{*}$ then $R(t) \rightarrow \infty$ as $t \rightarrow \infty$, whereas if $\beta^{*}<\beta<1-\mu_{1}$, then $R(t) \rightarrow 0$ as $t \rightarrow \infty$. 
The assertion $R(t) \rightarrow \infty$ as $t \rightarrow \infty$ is interpreted as the onset of cancer.

(ii') In case (ii) it was proved that there exist controllers $K\left(Q, \gamma_{1}\right)$ such that

$$
c \leq R(t) \leq C \text { for all } \mathrm{t}>0
$$

where $c, C$ are positive constants. This suggests that the spherical tumor remains within homeostatic bounds. Numerical simulations show that, in fact, $R(t)$ can be made to remain very close to a fixed radius $R(\infty)$.

Case (ii) means that if only TGF- $\beta$ and SMAD are mutated then the tumor remains benign.

The surface epithelium which bounds the intestinal lumen has the form described schematically in Figure 6.1.

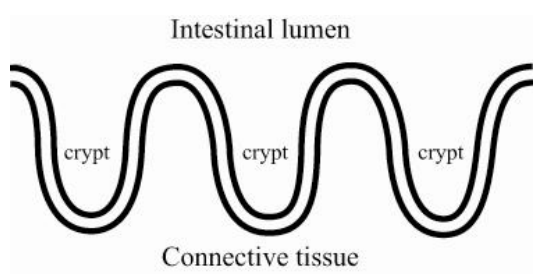

Figure 4: The shape of a normal colon.

Epithelial cells at the top of the crypt are in an environment of intensive chemical reactions and have a short life span of a few days. They are continuously replaced by new cells produced by stem cells which reside at the bottom of the crypt. A mechanistic model of colorectal cancer should therefore take into account the spatial geometry of the epithelium. A review article by van Leeuwen et al [68] describes various models of colorectal cancer; some of these models are compartmental, or lattice-based, while others consider stochastic methylation or genetic mutations. Mathematical models for early detection and treatment of colorectal cancer were introduced in [44]. Genetic instability in colorectal cancer was discussed in [56].

\section{Glioma}

Gliomas are characterized by their invasiveness into surrounding normal brain tissue, which leads to inevitable tumor recurrence after surgery. Patients diagnosed with glioblastoma, the most common and most aggressive of these tumors, have a median survival of approximately one year from the time of diagnosis. In order to improve patient outcome new therapeutic approaches are needed that target the invading cells. Several factors influence glioma cell migration in the brain. Migration can be stimulated by extracellular matrix (ECM) in a process known as haptotactic migration. Haptotaxis is enabled through pre-existing brain components, but is also greatly influenced by the ability of glioma cells themselves to modulate the surrounding ECM. Glioma cells remodel the surrounding ECM through the secretion of proteases, such as MMP, that are known to facilitate glioma invasion. Glioma cells motility is also stimulated by various molecules which they secrete, leading to chemotactic migration. These include ligands of the EGF family and the TGF- $\beta$ family. 
In patients, glioma cells usually follow preferred dispersion routes such as along white matter tracts and the basal lamina of brain blood vessels. This suggests that glioma cell migration may be dependent on specific substrates and structures in the brain. Indeed there are numerous publications based on a diffusion model for tumor cells in heterogeneous media for predicting invasion of glioma cells in the brain of patients [45] [59] [67]. A model consisting of a system of partial differential equations was developed by Eikenbery et al [17].

Here we describe a mathematical model, introduced by Kim et al [53], of glioma cells, in vitro, outside a spherical core, that is capable of reproducing the migration patterns observed in experiments, such as dispersion and branching. The model includes MMP and nutrient concentration, and takes into account the effects of chemotaxis, haptotaxis, and cell-cell adhesion.

We introduce the following variables:

$$
\begin{aligned}
& n=\text { glioma cells density } \\
& \rho=\text { ECM concentration, } \\
& P=\text { MMP concentration, } \\
& G=\text { nutrient concentration }
\end{aligned}
$$

The migration domain is a shell $\Omega=\left\{R_{0}<r<R_{1}\right\}$ surrounding the spherical tumor of fixed radius $R_{0}$. The following equations hold in $\Omega$ :

$$
\begin{aligned}
\frac{\partial n}{\partial t}= & \underbrace{D_{n} \Delta n}_{\text {dispersion }}-\underbrace{\nabla\left(\chi_{n} \frac{n \nabla G}{\sqrt{1+\lambda_{G}|\nabla G|^{2}}}\right)}_{\text {chemotaxis }} \\
& -\underbrace{\nabla\left(\chi_{n}^{1} \frac{n \nabla \rho}{\sqrt{1+\lambda_{\rho}|\nabla \rho|^{2}}}\right)}_{\text {haptotaxis }}-\underbrace{\nabla(n K(n))}_{\text {self-adhesion }}+\underbrace{\lambda_{11} n}_{\text {proliferation }}+\underbrace{S}_{\text {shedding }} \\
\frac{\partial \rho}{\partial t}= & -\underbrace{\lambda_{21} P \rho}_{\text {degradation }}+\underbrace{\lambda_{22} \rho\left(1-\frac{\rho}{\rho_{0}}\right)}_{\text {release/reconstruction }} \\
\frac{\partial P}{\partial t}= & \underbrace{D_{P} \Delta P}_{\text {Diffusion }}+\underbrace{\lambda_{31} n}_{\text {production by cells }}-\underbrace{\lambda_{32} P}_{\text {decay }} \\
\frac{\partial G}{\partial t}= & \underbrace{D_{G} \Delta G}_{\text {Diffusion }}-\underbrace{\lambda_{41} n \frac{G}{G+k_{G}}}_{\text {consumption }},
\end{aligned}
$$


Here $n(x, t) K(n(x, t))$ is the adhesive force acting on $n(x, t)$, where

$$
\begin{aligned}
K(n(x, t)) & =\frac{2}{\left|B_{\delta}(x)\right|} \int_{B_{\delta}(x)} \lambda_{a} f\left(n(y, t) \vec{S}_{x}(y) d y\right. \\
\vec{S}_{x}(y) & =\frac{y-x}{|y-x|} \\
f(n) & =k_{s} \frac{n}{n_{0}+n}
\end{aligned}
$$

and $k_{s}, n_{0}, \lambda_{a}, \eta$ are parameters that were estimated in [2] [66].

The shedding function $S$ is something unique to glioma. It describes how cancer cells are shed randomly from the surface of the tumor. In the 2-d case it is expressed numerically by introducing randomly in small regions $\left\{(r, \theta) ; R_{0}<r<R_{0}+\epsilon, \theta_{i-1}<\theta<\theta_{i}\right\}$ ( $\epsilon$ small $)$ at times $t_{j-1}<t<t_{j}$ new cancer cells with density equal to the density of tumor cells on the rim of the spherical tumor, $r=R_{0}$.

Simulation of the model shows that all the experimentally observed patterns can be obtained by varying three parameters: the chemotactic sensitivity $\chi_{n}$, the haptotactic coefficient $\chi_{n}^{1}$, and the adhesive force parameter $\lambda_{a}$. The model has been used in [53] to develop biologically testable hypotheses on slowing down the migration of glioma cells.

Advances in virology and tumor biology have enabled development of oncolytic viruses (OVs) which replicate selectively in tumor cells. OV progeny propagate throughout tumors, lysing tumor cells but not normal cells. Phases I and II clinical trials have shown OV therapy to be safe in some cancers but with limited efficacy; see [40] and references therein.

The standard treatment of glioma patients is surgery with follow-up radiation therapy. But there are currently ongoing experiments with OV therapy, in which a glioma slice is grafted to the brain of a mouse [40]. There is increasing evidence that the limited efficacy of the OV therapy is due to the host response to active viral infection: the innate immune system destroys the cancer cells infected by the virus, thereby decreasing the number of free virus, and the effectiveness of the OV therapy.

In order to block this counter-productive effect of the immune system, experiments have been conducted in which the immune suppressor cyclophosphamide was injected into the glioma. It was shown that this slowed down the growth of the tumor.

A corresponding mathematical model (based on [38] [69] [71]) was introduced in [39]. It uses the following variables:

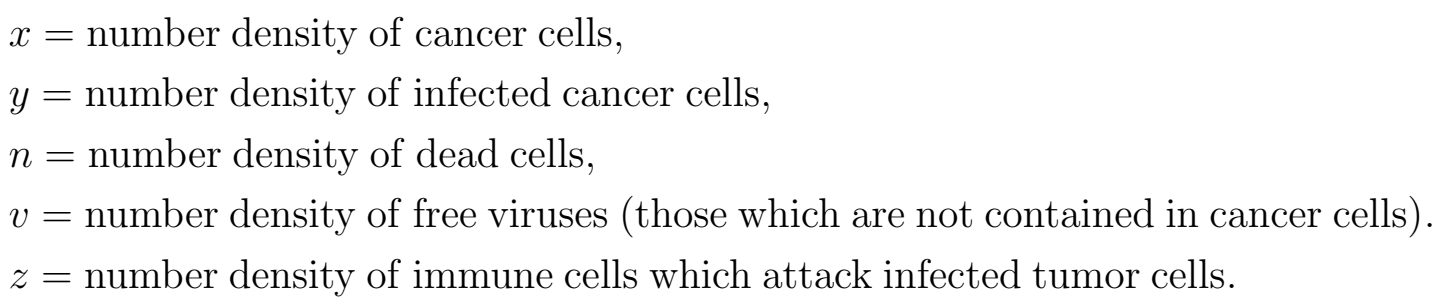


We assume that the tumor is radially symmetric and denote by $u(r, t)$ the radial velocity, $u(0, t)=$ 0 . Then, by conservation of number density, the following equations hold in the tumor region $\{r<R(t)\}$ :

$$
\begin{aligned}
& \frac{\partial x}{\partial t}+\frac{1}{r^{2}} \frac{\partial}{\partial r}\left(r^{2} u x\right)=\lambda x-\beta x v, \\
& \frac{\partial y}{\partial t}+\frac{1}{r^{2}} \frac{\partial}{\partial r}\left(r^{2} u y\right)=\beta x v-k y z-\delta y, \\
& \frac{\partial n}{\partial t}+\frac{1}{r^{2}} \frac{\partial}{\partial r}\left(r^{2} u n\right)=k y z-\omega z^{2}-\mu n, \\
& \frac{\partial z}{\partial t}+\frac{1}{r^{2}} \frac{\partial}{\partial r}\left(r^{2} u z\right)=s y z-\omega z^{2}-\mathrm{P}(t), \\
& \frac{\partial v}{\partial t}-D \frac{1}{r^{2}} \frac{\partial}{\partial r}\left(r^{2} \frac{\partial v}{\partial r}\right)=b \delta y-k_{0} v z-\gamma v .
\end{aligned}
$$

Here

$$
\begin{aligned}
& \lambda=\text { proliferation rate of tumor cells, } \\
& \beta=\text { infection rate, } \\
& \delta=\text { infected-cells lysis rate, } \\
& \mu=\text { removal rate of dead cells, } \\
& D=\text { dispersion coefficient of viruses, } \\
& b=\text { burst size of infected cells, } \\
& \gamma=\text { clearance rate of virus, } \\
& k=\text { immune killing rate, } \\
& k_{0}=\text { take-up rate of viruses, } \\
& s=\text { stimulation rate of infected cells, } \\
& \omega=\text { clearance rate of immune cells, }
\end{aligned}
$$

and $\mathrm{P}(t)$ is the concentration of cyclophosphamide. Some of the model parameters were estimated from the literature while others were obtained by fitting to experimental data from [40].

One of the model predictions is that if the burst size $b$ is larger than 100 then the radius of the cancer will decrease. For smaller values of $b($ e.g. $\mathrm{b}=50)$ the radius increases. The above mathematical model does not take into account side effects of the immune suppression; it also does not address the limitation on the dispersion of viruses caused by adhesive molecules in the ECM. 


\section{Prostate cancer}

The prostate is an accessory sex gland in the male reproductive system whose embryological development, growth, and function is dependent upon the primary male androgen, testosterone, that is primarily produced in the testes. Similarly, the etiology and progression of epithelial cancers arising in the prostate is strongly dependent upon the presence of testosterone. Testosterone, upon entering a prostate cell, is enzymatically converted to dihydrotestosterone (DHT) by the enzyme $5 \alpha$-reductase. Although testosterone and DHT both bind to and activate androgen receptor (AR), DHT has greater binding affinity and forms a more stable complex with AR than testosterone. Upon binding, the androgen-AR complex is phosphorylated and dimerizes followed by translocation to the cell nucleus where specific binding to the DNA elicits transcriptional activity of genes associated with growth, survival and proliferation.

The majority of patients are diagnosed with cancer considered localized to the prostate and are cured with primary treatment of surgery or radiation therapy. Yet, for those men who present with metastatic disease spread beyond the prostate or develop recurrence after local therapy, additional therapy targeting systemic disease is typically necessary. This therapy is based on drugs which act by different mechanisms; (i) inhibiting testicular testosterone production by down-regulation of gonadotropin-releasing hormone production by the pituitary, (ii) blocking the ability of testosterone to bind the androgen receptor, and (iii) introducing inhibition of the $5 \alpha$-reductase enzyme responsible for testosterone conversion to DHT.

Unfortunately, despite maintenance of castrate-levels of testosterone and inhibition of AR activity, eventually cancer cells $(\mathrm{CaP})$ develop resistance, generally first observed clinically as the rise of prostate-specific antigen (PSA) levels, followed by progressive disease. The rate of developing resistance is heterogeneous and may be observed within a few months, with an average of approximately two years. Clinical investigators are currently examining a role for intermittent, as opposed to continuous anti-androgen therapy, with goals of reducing the decrements in quality of life and perhaps having a therapeutic advantage of prolonging disease sensitivity to pharmacologic intervention. Intermittent treatment is accomplished by discontinuing anti-androgen therapy once a clinical objective or decline in PSA has been achieved, then restarting for disease progression, or a defined PSA threshold, or after a specific time period. This process would continue in cycles until resistance develops and the disease requires other interventions.

There are several mathematical models of serum dynamics in healthy prostate, $\mathrm{CaP}$ cell growth coupled to testosterone, DHT and AR dynamics [16] [63], and models which incorporate the interplay between cancer cells and mutated cancer cells [46] [47] [48].

Recently a more comprehensive model was developed by H. Jain et al [49]. It consists of a dynamical system for the following variables: 
$T_{2}=$ Serum testosterone concentration (in $\mathrm{nM}$ )

$P=$ Tissue PSA concentration (in $\mathrm{ng} / \mathrm{ml}$ )

$P_{S}=$ Serum PSA concentration (in $\mathrm{ng} / \mathrm{ml}$ )

$E=$ Normal prostatic epithelial cell density number (number of cells (in millions))

$N=$ Androgen-dependent cancer cell density number (number of cells (in millions))

$M=$ Castrate-resistant cancer cell density number (number of cells (in millions))

$T_{i}=$ Testosterone concentration in cell type $i, i=E, N, M$ (in nM)

$r_{i}^{0}=$ Constitutive free androgen receptor expression level in cell type $i$ (in nmoles per million cells)

$R_{i}=$ Free androgen receptor concentration in cell type $i$ (in $\mathrm{nM}$ )

$D_{i}=$ DHT concentration in cell type $i$ (in $\mathrm{nM}$ )

$A_{i}=$ DHT-activated androgen receptor concentration in cell type $i$ (in $\mathrm{nM}$ )

$A_{i t}=$ Testosterone-activated androgen receptor concentration in cell type $i$ (in $\mathrm{nM}$ )

The differential equations for these variables are based on the following scheme. Testosterone is synthesized by the testes and released into the blood stream. It then enters the prostate and is taken up by prostatic epithelial cells $(E)$, androgen-dependent cancer cells $(N)$, and castrateresistant cancer cells $(M)$. Within the cells, it is converted into DHT by the action of $5 \alpha$-reductase. Both testosterone and DHT subsequently activate androgen receptors, resulting in proliferative and survival signal modulation, together with PSA release into the tissue, from where it extravasates into the blood stream. The intracellular signaling pathway for $E$ cells is similar to the of $N$ cells. Growth factors expressed by stromal cells in response to testosterone signaling such as VEGF and TGF- $\beta$ may also contribute to epithelial cell proliferation and survival. The model includes mutations resulting in castrate-resistance and the actions of the three types of drugs mentioned above. The equations governing the rate of change of tissue PSA and serum PSA are

$$
\begin{aligned}
& \frac{d P}{d t}=-\alpha_{P} P E_{f r a c}-\gamma_{P} P \frac{(N+M)^{2}}{K_{P}+N+M}-\lambda_{P} P+\sum_{i=E, N, M} \beta_{P_{i}}\left(A_{i}+A_{i t}+\rho_{i}\right) i \\
& \frac{d P_{S}}{d t}=\alpha_{P} P E_{f r a c}+\gamma_{P} P \frac{(N+M)^{2}}{K_{P}+N+M}-\lambda_{P_{S}} P_{S}
\end{aligned}
$$

where $E_{\text {frac }}$ is the epithelial and $\mathrm{CaP}$ cell fraction in prostate, and all other parameters are constants.

The parameters introduced in the dynamical system of Jain et al [49] can be divided into two groups: (i) Parameters whose values have been determined either directly from the experimental literature, or by data fitting, and (ii) Personalized parameters. The personalized parameters are the rate of acquisition of new mutations conferring androgen resistance, competitive advantage of hormone sensitive cells over hormone refractory, and overall sensitivity to androgen deprivation. These parameters may vary from one cancer patient to another, and they reflect the deep hetrogeneity of the disease. Numerical simulations show how these personalized parameters can be 
determined to achieve the same prognostics, in terms of PSA and Cap concentration, for different groups of patients as reported in the literature. Relating patient histories to the personalized parameters may suggest guidelines for personalized progress under different therapy scheduling strategies, which underscores the potential of the model as developing into a valuable tool to aid clinicians in making treatment choices.

\section{Other cancers}

We briefly review several other specific cancers for which mathematical models have been developed.

\subsection{Melanoma}

In recent work, Eikenberry et al [18] [19] developed a mathematical model of melanoma. The model is formulated by a system of PDEs which include the following variables: cancer and healthy epithelial cells, necrotic debris, TAF, endothelial cells, oxygen pressure and basement membrane density; the membrane separates the skin surface from the vascular system, and angiogenesis is initiated once the cancer size has become large enough. The model also includes the immune response by macrophages and killer cells (NKs) and immune attracting factor (IAF).

The model predicts that the immune response to the primary tumor can destroy or hold in check the vast majority of metastases that are produced. Clinical data [41] suggest a delicate tumorimmune balance slightly in favor of the tumor, although, currently, immunostimulatory Interferon$\alpha$ and IL-2 are approved as adjuvant therapy for melanoma.

\subsection{Bladder cancer}

Immunotherapy with Bacillus Calmette-Gurin (BCG) is a clinically established procedure for the treatment of superficial bladder cancer. Bunimovich-Mendrazitsky et al [7] [8] developed an ODE model of BCG therapy. The model predicts that the intensity of immunotherapy must not exceed certain bounds which depend on the initial size of the tumor.

\subsection{Pancreatic cancer}

Statistical studies show that metastasis of pancreatic cancer occurs late during genetic evolution [1]. In fact, progress to lethal stage takes at least 15 years. Once one cancer cell grows and has the ability to spread to other tissues, death occurs within 2-3 years. This situation suggests that early screening for pancreatic cancer would be extremely beneficial. Hence there is a need to identify the initial set of mutations that leads to progression to cancer. What is known is that detection of mitochondrial DNA mutations in pancreatic cancer offers a significant advantage over detection of nuclear DNA mutation [51]. But there is presently no mathematical models of mechanistic approach to detection of pancreatic cancer progression. 


\section{Conclusion}

In this review we first described some common features of solid tumors, and then proceeded to describe mathematical models of several specific cancers, each with its own particular set of issues. For a mathematical model to be useful to biologists the model must first of all predict results that are in agreement with what is already known to the biologists. But it must also go further and suggest hypotheses that are biologically testable. Of course not all mathematical models satisfy one or both requirements; this is to be expected, especially when the biological process is highly complex, as is often the case with cancer progression. In such cases even a model which establishes just a 'proof of concept' is a worthwhile contribution.

An example is liver cancer. Here a Gompretzian exponential growth was introduced in [10] which describes the tumor growth prior to treatment by radiation; the model incorporates biological cancer markers; more progress could be made by using models of liver cell aggregation [42] [43].

In the case of lung cancer, the leading cause of death among all forms of cancer, the only studies so far are statistical models that inform the effect of smoking on cancer occurrance, and the effect of lung cancer screening on reducing deaths.

There are many challenges ahead in modeling these cancers.

\section{References}

[1] A. Angelle. Pancreatic cancer shown to be surprisingly slow killer. Live Science, October $27,2010$.

[2] N. Armstrong, K. Painter, J. Sherratt. A continuum approach to modeling cell-cell adhesion. J. Theor. Biol., 243 (1), 98-113.

[3] B.P. Ayati, G.F. Webb, A.R.A. Anderson. Computational methods and results for structured multiscale methods of tumor invasion. Multiscale Model. Simul., 5 (2006), 1-20.

[4] S. Aznavoorian, M. Stracke, H. Krutzsch, E. Schiffmann, L. Liotta. Signal transduction for chemotaxis and haptotaxis by matrix molecules in tumor cells. J. Cell Biol., 110(4), (1990), 1427-1438.

[5] B. Bazaliy, A. Friedman. Global existence and stability for an elliptic-parabolic free boundary problem: Application to a model with tumor growth. Indiana Univ. Math. J., 52 (2003), 1265-1304.

[6] B.V. Bazaliy, A. Friedman. A free boundary problem for an elliptic-parabolic system: Application to a model of tumor growth. Comm. in PDE, 28 (2003), 627.

[7] S. Bunimovich-Mendrazitsky, E. Shochat, L. Stone. Mathematical Model of BCG immunotherapy in superficial bladder cancer. Bull. Math. Biol., 69 (2007), 1847-1870. 
[8] S. Bunimovich-Mendrazitsky, J.C. Gluckman, J. Chaskalovich. A mathematical model of combined bacillus Calmette-Guerin (BCG) and interleuken (IL)-2 immunotherapy of superficial bladder cancer. J. Theor. Biol, 277 (2011), 27-40.

[9] H.M. Byrne, M.A.J. Chaplain. Growth of necrotic tumors in the presence and absence of inhibitors. Math. Biosci., 135 (1996), 187-216.

[10] A. Campbell, T. Sivakumaran, M. Davidson, M. Lock, E. Wong. Mathematical modeling of liver metastases tumour growth and control with radiotherapy. Phys. Med. Biol., 53 (2008), 7225-7239.

[11] X. Chen, A. Friedman. A free boundary problem for elliptic-hyperbolic system: An application to tumor growth. SIAM J. Math. Anal., 35 (2003), 974-986.

[12] X. Chen, S. Cui, A. Friedman. A hyperbolic free boundary problem modeling tumor growth: Asymptotic behavior. Trans. Amer. Math. Soc., 357 (2005), 4771-4804.

[13] S. Cui, A. Friedman. Analysis of a mathematical model of the growth of necrotic tumors. J. Math. Anal. \& Appl., 255 (2001), 636-677.

[14] S. Cui, A. Friedman. A free boundary problem for a singular system of differential equations: An application to a model of tumor growth. Trans. Amer. Math. Soc., 355 (2003), 3537-3590.

[15] S. Cui, A. Friedman. A hyperbolic free boundary problem modeling tumor growth. Interfaces \& Free Boundaries, 5 (2003), 159-182.

[16] S.E. Eikenberry, J.D. Nagy, Y. Kuang. The evolutionary impact of androgen levels on prostate cancer in a multi-scale mathematical model. Biol. Direct, 5 (2010), 24-52.

[17] S.E. Eikenberry, T. Sankar, M.C. Preul, E.J. Kostelich, C.J. Thalhauser, Y. Kuang. Virtual glioblastoma: growth, migration and treatment in a three-dimensional mathematical model. Cell Prolif., 42 (2009), 511-528.

[18] S. Eikenberry, C. Thalhauser, Y. Kuang. Mathematical modeling of melanoma. PLoS Comput Biol., 5:e1000362 (2009).

[19] S. Eikenberry, C. Thalhauser, Y. Kuang. Tumor-immune interaction, surgical treatment, and cancer recurrence in a mathematical model of melanoma. PLoS Comput Biol., 5:e1000362 (2009), Epub 2009, April 24.

[20] M.A. Fontelos, A. Friedman. Symmetry-breaking bifurcations of free boundary problems in three dimensions. Asymptotic Anal., 35 (2003), 187-206.

[21] S.J.H. Franks, H.M. Byrne, J.P. King, J.C.E. Underwood, C.E. Lewis. Modeling the early growth of ductal carcinoma in situ of the breast. J. Math. Biol., 47 (2003), 424-452. 
[22] S.J.H. Franks, H.M. Byrne, J.P. King, J.C.E. Underwood, C.E. Lewis. Modeling the growth of comedo ductal carcinoma in situ. Math. Med. \& Biol., 20 (2003), 277-308.

[23] S.J.H. Franks, H.M. Byrne, J.C.E. Underwood, C.E. Lewis. Biological inferences from a mathematical model of comedo ductal carcinoma in situ of the breast. J. Theor. Biol., 232 (2005), 523-543.

[24] S.J.H. Franks, J.P. King. Interactions between a uniformly proliferating tumor and its surroundings: Uniform material properties. Math. Med. \& Biol., 20 (2003), 47-89.

[25] A. Friedman. A free boundary problem for a coupled system of elliptic, hyperbolic, and Stokes equations modeling tumor growth. Interfaces and Free Boundaries, 8 (2006), 247-261.

[26] A. Friedman. A multiscale tumor model. Interfaces \& Free Boundaries, 10 (2008), 245-262.

[27] A. Friedman. Free boundary value problems associated with multiscale tumor models. Mathematical Modeling of Natural Phenomena, 4 (2009), 134-155.

[28] A. Friedman, B. Hu. Bifurcation from stability to instability for a free boundary problem arising in tumor model. Arch. Rat. Mech. Anal., 180 (2006), 293-330.

[29] A. Friedman, B. Hu. Asymptotic stability for a free boundary problem arising in a tumor model. J. Diff. Eqs., 227 (2006), 598-639.

[30] A. Friedman, B. Hu. Bifurcation from stability to instability for a free boundary problem modeling tumor growth by Stokes equation. Math. Anal \& Appl., 327 (2007), 643-664.

[31] A. Friedman, B. Hu. Bifurcation for a free boundary problem modeling tumor growth by Stokes equation. SIAM J. Math. Anal., 39 (2007), 174-194.

[32] A. Friedman, B. Hu. Stability and instability of Liapounov-Schmidt and Hopf bifurcations for a free boundary problem arising in a tumor model. Trans. Amer. Math. Soc., 360 (2008), 5291-5342.

[33] A. Friedman, B. Hu. The role of oxygen in tissue maintenance: Mathematical modeling and qualitative analysis. Math. Mod. Meth. Appl. Sci., 18 (2008), 1-33.

[34] A. Friedman, B. Hu, C-Y. Kao. Cell cycle control at the first restriction point and its effect on tissue growth. J. Math. Biol., 60 (2010), 881-907.

[35] A. Friedman, Y. Kim. Tumor cells-proliferation and migration under the influence of their microenvironment. Math Biosci. \& Engin., 8 (2011), 373-385.

[36] A. Friedman, F. Reitich. Analysis of a mathematical model for growth of tumors. J. Math. Biol., 38 (1999), 262-284. 
[37] A. Friedman, F. Reitich. Symmetry-breaking bifurcation of analytic solutions to free boundary problems: An application to a model of tumor growth. Trans. Amer. Math. Soc., 353 (2001), 1587-1634.

[38] A. Friedman, Y. Tao. Analysis of a model of virus that replicates selectively in tumor cells. J. Math. Biol., 47 (2003), 391-423.

[39] A. Friedman, J.J. Tian, G. Fulci, E.A. Chiocca, J. Wang. Glioma virotherapy: The effects of innate immune suppression and increased viral replication capacity. Cancer Research, 66 (2006), 2314-2319.

[40] G. Fulci, L. Breymann, D. Gianni, K. Kurozomi, S. Rhee, J. Yu, B. Kaur, D. Louis, R. Weissleder, M. Caligiuri, E.A. Chiocca. Cyclophosphamide enhances glioma virotherapy by inhibiting innate immune responses. PNAS, 103 (2006), 12873-12878.

[41] V. DeGiorgi, D. Massai, G. Gerlini, F. Mannone, E. Quercioli, et al. Immediate local and regional recurrence after the excision of a polypoid melanoma: Tumor dormancy or tumor activation. Dermatol. Surg., 29 (2003), 664-667.

[42] J.E.F. Green, S.L. Waters, K.M. Shakesheff, H.M. Byrne. A Mathematical Model of Liver Cell Aggregation In Vitro. Bull. Math. Biol., 71 (2009), 906-930.

[43] J.E.F. Green, S.L. Waters, J.P. Whiteley, L. Edelstein-Keshet, K.M. Shakesheff, H.M. Byrne. Nonlocal models for the formation of hepatocyte-stellate cell aggregates. J. Theor. Biol., 267 (2010), 106-120.

[44] P.R. Harper, S.K. Jones. Mathematical models for the early detection and treatment of colorectal cancer. Health Care Management Science, 8 (2005), 101-109.

[45] H. Harpold, J. Ec, K. Swanson. The evolution of mathematical modeling of glioma proliferation and invasion. J. Neuropathol. Exp. Neurol., 66 (1) (2007), 1-9.

[46] A. Ideta, G. Tanaka, T. Takeuchi, K. Aihara. A Mathematical model of intermittent androgen suppression for prostate cancer. J. Nonlinear Sci., 18 (2008), 593-614.

[47] T.L. Jackson. A mathematical model of prostate tumor growth and androgen-independent relapse. Discrete Cont. Dyn-B, 4 (2004), 187-201.

[48] T.L. Jackson. A mathematical investigation of the multiple pathways to recurrent prostate cancer: comparison with experimental data. Neoplasia, 6 (2004), 697-704.

[49] H.V. Jain, S. Clinton, A. Bhinder, A. Friedman. Mathematical model of hormone treatment for prostate cancer, to appear.

[50] Y. Jiang, J. Pjesivac-Grbovic, C. Cantrell, J.P. Freyer. A multiscale model for avascular tumor growth. Biophy. J., 89 (2005), 3884-3894. 
[51] J.B. Jones, J.J. Song, P.M. Hempen, G. Parmigiani, R.H. Hruban, S.E. Kern. Detection of mitochondrial DNA mutations in pancreatic cancer offers a "Mass"-ive advantage over detection of nuclear DNA mutations. Cancer Research, 61 (2001), 1299-1304.

[52] Y. Kim, A. Friedman. Interaction of tumor with its microenvironment: a mathematical model. Bull. Math. Biol., 72 (2010), 1029-1068.

[53] Y. Kim, S. Lawler, M.O. Nowicki, E.A. Chiocca, A. Friedman. A mathematical model of brain tumor: pattern formation of glioma cells outside the tumor spheroid core. J. Theor. Biol., 260 (2009), 359-371.

[54] Y. Kim, M. Stolarska, H. Othmer. A hybrid model for tumor spheroid growth in vitro I: theoretical development and early results. Math. Mod. Meth. Appl. Sci., 17 (2007), 17731798.

[55] Y. Kim, J. Wallace, F. Li, M. Ostrowski, A. Friedman. Transformed epithelial cells and fibroblasts/myofibroblasts interaction in breast tumor: a mathematical model and experiments. J. Math. Biol., 61 (2010), 401-421.

[56] N.L. Komarova, C. Lengauer, B. Vogelstein, M. Nowak. Dynamics of genetic instability in sporadic and familial colorectal cancer. Cancer Biology \& Therapy, 1 (2002), 685-692.

[57] H.A. Levine, M. Nilsen-Hamilton. Angiogenesis-A biochemical/mathematical perspective. Lecture Notes Math., 1872 (2006), 23-76, Springer-Verlag, Berlin-Heidelberg.

[58] H.A. Levine, S.L. Pamuk, B.D. Sleeman, M. Nilsen-Hamilton. Mathematical modeling of capillary formation and development in tumor angiogenesis: penetration into the stroma. Bull. Math. Biol., 63 (2001), 801-863.

[59] E. Mandonnet, J. Delattre, M. Tanguy, K. Swanson, A. Carpentier, H. Duffau, P. Cornu, R. Effenterre, J. Ec, L.J. Capelle. Continuous growth of mean tumor diameter in a subset of grade ii gliomas. Ann. Neurol., 53 (4) (2003), 524-528.

[60] N. Mantzaris, S. Webb, H.G. Othmer. Mathematical modeling of tumor angiogenesis. J. Math. Biol., 49 (2004), 111-187.

[61] A. Perumpanani, H. Byrne. Extracellular matrix concentration exerts selection pressure on invasive cells. Eur. J. Cancer, 35(8) (1999), 1274-1280.

[62] G.J. Pettet, C.P. Please, M.J. Tindall, D.L.S. McElwain. The migration of cells in multicell tumor spheroids. Bull. Math. Biol., 63 (2001), 231-257.

[63] L.K. Potter, M.G. Zagar, H.A. Barton. Mathematical model for the androgenic regulation of the prostate in intact and castrated adult male rats. Am. J. Physiol. Endocrinol. Metab., 291 (2006), E952-E964. 
[64] R. Ribba, T. Colin, S. Schnell. A multiscale model of cancer, and its use in analyzing irradiation therapies. Theor. Biol. \& Med. Mod., 3 (2006), No. 7, 1-19.

[65] B. Ribba, O. Sant, T. Colin, D. Bresch, E. Grenien, J.P. Boissel. A multiscale model of avascular tumor growth to investigate agents. J. Theor. Biol., 243 (2006), 532-541.

[66] J. Sherratt, S. Gourley, N. Armstrong, K. Painter. Boundedness of solutions of a non-local reaction diffusion model for adhesion in cell aggregation and cancer invasion. Eur. J.Appl. Math., 20 (2009), 123-144.

[67] K. Swanson, J. Ec, J. Murray. A quantitative model for differential motility of gliomas in grey and white matter. Cell Prolif., 33 (5) (2000), 317-329.

[68] I.M.M. van Leeuwen, H.M. Byrne, O.E. Jensen, J.R. King. Crypt dynamics and colorectal cancer: advances in mathematical modeling. Cell Prolif., 39 (2006), 157-181.

[69] J.T. Wu, H.M. Byrne, D.H. Kirn, L.M. Wein. Modeling and analysis of a virus that replicates selectively in tumor cells. Bull. Math. Biol., 63 (2001), 731-768.

[70] J. Wu, S. Cui. Asymptotic stability of stationary solutions of a free boundary problem modeling the growth of tumors with fluid tissues. SIAM J. Math. Anal., 41 (2010), 391-414.

[71] J.T. Wu, D.H. Kirn, L.M. Wein. Analysis of a three-way race between tumor growth, a replication-competent virus and an immune response. Bull. Math. Biol., 66 (2004), 605-625. 